\title{
Une revue sur quelques inégalités fonctionnelles et les propriétés de symétrie pour leurs fonctions extrémales
}

\author{
Maria J. Esteban \\ CEREMADE (UMR CNRS 7534) \\ Université Paris-Dauphine \\ F-75775 Paris Cedex 16 \\ E-mail: esteban@ceremade.dauphine.fr
}

(d'après des travaux en collaboration avec J. Dolbeault, M. Loss, G. Tarantello and A. Tertikas)

\begin{abstract}
Cette revue est la version écrite d'un exposé sur quelques résultats concernant les propriétés de symétrie des fonctions extremales pour les inégalités de caffarelli-Kohn-Nireberg
\end{abstract}

\section{Remarques générales.}

Dans un cadre général, on est intéressé par l'existence d'une constante $C>0$ telle que dans un certain espace fonctionnel $X$, pour toute $u \in X$,

$$
F(D u, u, x) \leq C G\left(D^{2} u, D u, u, x\right) .
$$

Les inégalités fonctionnelles jouent un rôle important dans l'obtention d'estimations a priori pour les solutions d'EDPs, dans l'analyse du comportement en temps long des solutions de problèmes d'évolution et dans la description du profil d'explosion dans des cas d'explosion des solutions en temps fini d'équations paraboliques (chaleur, Schrödinger, etc). Et aussi pour de multiples questions reliées au transport optimal, en analyse, mécanique, géométrie, etc.

La connaissance de la valeur précise de $C$ ou des propriétés qualitatives des solutions (quand elles existent) est cruciale dans pas mal des cas cités ci-dessus. 
Questions importantes :

- Est la constante $C$ atteinte dans $X$ ?

- Si oui, comment sont les solutions $u$ ?

Remarque. Si nous savons a priori que les solutions extrémales ont certaines propriétés de symmetrie, par exemple si elles sont radiales, etc., il sera alors plus facile de calculer la valeur de $C$.

\section{Inégalités de Caffarelli-Kohn-Nirenberg (CKN).}

Les inégalités suivantes ont été étudiées sytématiquement pour la première fois dans [4].

$$
\left(\int_{\mathbb{R}^{d}} \frac{|u|^{p}}{|x|^{b p}} d x\right)^{2 / p} \leq \mathrm{C}_{a, b} \int_{\mathbb{R}^{d}} \frac{|\nabla u|^{2}}{|x|^{2 a}} d x \quad \forall u \in \mathcal{D}_{a, b},
$$

avec $a \leq b \leq a+1$ if $d \geq 3, a<b \leq a+1$ si $d=2$, et $a \neq \frac{d-2}{2}$,

$$
p=\frac{2 d}{d-2+2(b-a)}
$$

$\mathcal{D}_{a, b}:=\left\{|x|^{-b} u \in L^{p}\left(\mathbb{R}^{d}, d x\right):|x|^{-a}|\nabla u| \in L^{2}\left(\mathbb{R}^{d}, d x\right)\right\}$.

Notons que :

$$
\begin{gathered}
b-a \rightarrow 0 \Longleftrightarrow p \rightarrow \frac{2 d}{d-2}, \\
b-(a+1) \rightarrow 0 \Longleftrightarrow p \rightarrow 2_{+}, \\
\frac{1}{C_{a, b}}=\inf _{\mathcal{D}_{a, b}} \frac{\int_{\mathbb{R}^{d}} \frac{|\nabla u|^{2}}{|x|^{2 a}} d x}{\left(\int_{\mathbb{R}^{d}} \mid \frac{|u|^{p}}{|x|^{b p}} d x\right)^{2 / p}} .
\end{gathered}
$$

\section{La question de la symétrie.}

Définissons les constantes optimales pour le cas symétrique et pour le cas général :

$\mathrm{C}_{a, b}:=$ meilleure constante pour des fonctions générales $u$, 
$\mathrm{C}_{a, b}^{*}:=$ meilleure constante pour des fonctions symétriques $u$.

Alors, on a : $\mathrm{C}_{a, b}^{*} \leq C_{a, b}$, et à une dilatation et multiplication par une constante près, la fonction optimale est

$$
u_{a, b}^{*}(x)=\left(1+|x|^{-\frac{2 a(1+a-b)}{b-a}}\right)^{-\frac{b-a}{1+a-b}} .
$$

Questions: A-t-on $u_{a, b}=u_{a, b}^{*}$ ? Et si pas toujours, pour quelles valeurs de $a$ et $b$ ?

Résultats connus (L. Caffarelli- R. Kohn-L. Nirenberg [4], Th. Aubin [1], G. Talenti [21], E.H. Lieb [17], K.S. Chou-C.W. Chu [6], F. Catrina-Z.Q. Wang $[5], \ldots)$.

- Existence de solutions extrémales dans la demie-bande $a<b<a+1$, $a<\frac{d-2}{2}$.

- Symétrie (et existence) dans la zone $a \leq b<a+1,0<a<\frac{d-2}{2}$.

- Non-existence de solutions extrémales pour $a<0$ et $b=a$ ou $b=$ $a+1$.

\section{Symétrie et rupture de symétrie. Résultats ultérieurs.}

RUPTURE DE SYMETRIE : F. Catrina-Z.Q. Wang [5], V. Felli-Schneider [12], dans certaines régions de la zone $a<0$ : pour $b \in(a, a+A), b<b^{F S}(a)$, avec

$$
b^{F S}(a)=\frac{d(d-2-2 a)}{2 \sqrt{(d-2-2 a)^{2}+4(d-1)}}-\frac{1}{2}(d-2-2 a) .
$$

SYMETRIE : C.S. Lin, Z.Q. Wang [18]; J. Dolbeault, M.J. Esteban, G. Tarantello $(\mathrm{d}=2)$ [10] (pour $a<0$, dans un voisinage de $a=0$ ).

SYMETRIE : J. Dolbeault, M.J. Esteban, M. Loss, G. Tarantello [9] (dans un voisinage de $b=a+1$ ).

\section{Transformation d'Emden-Fowler et le cylindre $\mathcal{C}=\mathbb{R} \times S^{d-1}$.}

Le changement de variables et de fonctions suivant permettent d'éliminer les poids présents dans les inégalités de Caffarelli-Kohn-Nirenberg, qui dans 
les nouvelles variables s'écrivent comme des inégalités dans un cylindre infini

$$
t=\log |x|, \quad \theta=\frac{x}{|x|} \in S^{d-1}, \quad w(t, \theta)=|x|^{-a} v(x), \quad \Lambda=\frac{1}{4}(d-2-2 a)^{2} .
$$

- Les inégalités de Caffarelli-Kohn-Nirenberg re-écrites dans le cylindre deviennent des inégalités d'interpolation du type Gagliardo-Nirenberg :

$$
\begin{gathered}
\|w\|_{L^{p}(\mathcal{C})}^{2} \leq C_{\Lambda, p}\left[\|\nabla w\|_{L^{2}(\mathcal{C})}^{2}+\Lambda\|w\|_{L^{2}(\mathcal{C})}^{2}\right], \\
\text { où } \quad \mathcal{E}_{\Lambda}[w]:=\|\nabla w\|_{L^{2}(\mathcal{C})}^{2}+\Lambda\|w\|_{L^{2}(\mathcal{C})}^{2},
\end{gathered}
$$

et alors,

$$
C_{\Lambda, p}^{-1}:=\mathrm{C}_{a, b}^{-1}=\inf \left\{\mathcal{E}_{\Lambda}(w):\|w\|_{L^{p}(\mathcal{C})}^{2}=1\right\} .
$$

Dans le cylindre il est plus aisé de travailler avec les paramètres $(\Lambda, p)$ qu'avec $(a, b)$. Remarquons par ailleurs la relation entre les contraintes imposées à $a$ et celles trouvées pour $\Lambda$, et la définition de la courbe de FelliSchneider $b=b^{F S}(a)$ dans le choix des paramètres $(\Lambda, p)$ :

$$
\begin{gathered}
a<\frac{d-2}{2} \Longrightarrow \Lambda>0, \quad a<0 \Longrightarrow \Lambda>\frac{1}{4}(d-2)^{2}, \\
b<b^{F S}(a) \quad \Longleftrightarrow \quad \Lambda>\Lambda_{F S}(p):=\frac{4(d-1)}{p^{2}-1} .
\end{gathered}
$$

\section{Stratégies pour les démonstrations.}

- Existence : par méthode de minimisation + utilisation classique de la méthode de concentration-compacité $([19,20,5])$.

- Symétrie $a>0$ : par "moving-planes" ou symétrisation $([1,21,2,13$, $17,14,11])$.

- Symétrie $a<0$ proche de 0 : par une une métode perturbative ([18]), singulière dans le cas $d=2([10])$.

- Symétrie $b$ proche de $a+1$ : par une étude fine du problème linéarisé dans cette zone + étude du spectre de l'opérateur linéarisé [9]. 
Rupture de symétrie : en étudiant la fonctionnelle dans un voisinage des solutions extrémales radiales. On trouve en fait que pour $(a, b)$ tel que $b<b^{F S}(a)$, ou de manière équivalente, $\Lambda>\Lambda_{F S}(p)$; ces fonctions sont instables dans l'espace $(\mathcal{C})$ :

$$
\mathcal{E}^{\prime \prime}\left(u_{\Lambda, p}^{*}\right)(w)=\left(|a(\Lambda, p)| p-\left(1+|a(\Lambda, p)|^{2}\right)+\cdots\right)\|w\|_{L^{2}}^{2}<0
$$

quand $\Lambda>\Lambda_{F S}(p)$ and $w$ est orthogonale à toutes les fonctions qui ne dépendent pas des variables angulaires $\theta$. Voir le travail de Catrina and Wang [5] pour un premier résultat, et celui de Felli and Schneider [12] pour un résultat complet.

Ceci se fait en étudiant le spectre de l'opérateur

$$
-\Delta+\Lambda-(p-1)\left|w_{\Lambda, p}^{*}\right|^{p-2}
$$

dans $H^{1}(\mathcal{C})$ et après on utilise la décomposition en harmoniques sphériques.

Deux régions simplement connexes séparées par une courbe continue.

Dans [9] il est montré que les zones de symétrie et de rupture de symétrie sont simplement connexes et séparées par une courbe continue, $\Lambda=\bar{\Lambda}(p)$.

Question ouverte : La courbe de Felli-Schneider, définie par $b=$ $b^{F S}(a)$ (ou de manière équivalente, par $\Lambda=\Lambda_{F S}(p)$ ) et celle ci-dessus coïncident-elles ? Dans la suite nous répondons partialement à cette question.

Idée de la démonstration. Changements d'échelle et conséquences.

Soit $w_{\sigma}(t, \theta):=w(\sigma t, \theta)$ pour $\sigma>0$. On démontre facilement que :

$$
\mathcal{F}_{\sigma^{2} \Lambda, p}\left(w_{\sigma}\right)=\sigma^{1+2 / p} \mathcal{F}_{\Lambda, p}(w)-\sigma^{-1+2 / p}\left(\sigma^{2}-1\right) \frac{\int_{\mathcal{C}}\left|\nabla_{\theta} w\right|^{2} d y}{\left(\int_{\mathcal{C}}|w|^{p} d y\right)^{2 / p}}
$$

L'utilisation de la formule ci-dessus permet de montrer facilement l'existence de la courbe séparant les zones de symétrie et de rupture de symétrie. La semi-continuité supérieure de la courbe $p \rightarrow \bar{\Lambda}(p)$ se démontre facilement. Pour prouver la continuité, il faut une analyse spectrale délicate.

CONJECTURE: $\bar{\Lambda}(p)$ coïncide avec $\Lambda_{F S}(p)$. 


\section{Inégalités généralisées de Caffarelli-Kohn-Nirenberg inequalities (CKN) .}

Les inégalités suivantes ont été discutées pour la première fois dans [4]. Voir un travail plus récent et très complet sur ces inégalités dans [7].

Soit $d \geq 3$. Pour tout $p \in\left[2, p(\theta, d):=\frac{2 d}{d-2 \theta}\right]$, il existe une constante positive $\mathrm{C}(\theta, p, a)$ telle que

$$
\left(\int_{\mathbb{R}^{d}} \frac{|u|^{p}}{|x|^{b p}} d x\right)^{\frac{2}{p}} \leq \mathrm{C}(\theta, p, a)\left(\int_{\mathbb{R}^{d}} \frac{|\nabla u|^{2}}{|x|^{2 a}} d x\right)^{\theta}\left(\int_{\mathbb{R}^{d}} \frac{|u|^{2}}{|x|^{2(a+1)}} d x\right)^{1-\theta} .
$$

Dans le cas radial, avec $\Lambda=\left(a-\frac{d-2}{2}\right)^{2}$, la meilleure constante radiale est $C_{C K N}^{*}(\theta, p, a)$ and (see [7] :

$$
\begin{gathered}
C_{C K N}(\theta, p, a) \geq C_{C K N}^{*}(\theta, p, a)=C_{C K N}^{*}(\theta, p) \Lambda^{\frac{p-2}{2 p}-\theta} \\
C_{C K N}^{*}(\theta, p)=\left[\frac{2 \pi^{d / 2}}{\Gamma(d / 2)}\right]^{2 \frac{p-1}{p}}\left[\frac{(p-2)^{2}}{2+(2 \theta-1) p}\right]^{\frac{p-2}{2 p}}\left[\frac{2+(2 \theta-1) p}{2 p \theta}\right]^{\theta}\left[\frac{4}{p+2}\right]^{\frac{6-p}{2 p}}\left[\frac{\Gamma\left(\frac{2}{p-2}+\frac{1}{2}\right)}{\sqrt{\pi} \Gamma\left(\frac{2}{p-2}\right)}\right]^{\frac{p-2}{p}} .
\end{gathered}
$$

\section{Stratégie des démonstrations.}

Comme dans le cas $\theta=1$, des ingrédients importants sont la transformation d'Emden-Fowler et la minimisation dans le cylindre $\mathcal{C}$ de la fonctionnelle

$$
\mathcal{E}_{\theta}[v]:=\left(\|\nabla v\|_{\mathrm{L}^{2}(\mathcal{C})}^{2}+\Lambda\|v\|_{\mathrm{L}^{2}(\mathcal{C})}^{2}\right)^{\theta}\|v\|_{\mathrm{L}^{2}(\mathcal{C})}^{2(1-\theta)}
$$

sous la contrainte $\|v\|_{\mathrm{L}^{p}(\mathcal{C})}=1$.

Existence : Convergence des suites minimisantes si elles sont bornées dans $H^{1}(\mathcal{C})$ : par une utilisation plus ou moins classique de la méthode de concentration-compacité..

Pour $p \in(2, p(\theta, d))$, l'existence se démontre facilement pour tout $\Lambda>0$.

Pour $p=p(\theta, d)$, les estimationsa priori peuvent être obtenues par une série d'inégalités entre les différentes termes de l'énergie du problème.

\section{Une possible perte de compacité.}


- Les inégalités d' interpolation de Gagliardo-Nirenberg : si $p \in\left(2,2^{*}\right)$,

$$
\|u\|_{\mathrm{L}^{p}\left(\mathbb{R}^{d}\right)}^{2} \leq C_{G N}(p)\|\nabla u\|_{\mathrm{L}^{2}\left(\mathbb{R}^{d}\right)}^{2 \vartheta(p, d)}\|u\|_{\mathrm{L}^{2}\left(\mathbb{R}^{d}\right)}^{2(1-\vartheta(p, d))} \quad \forall u \in \mathrm{H}^{1}\left(\mathbb{R}^{d}\right) .
$$

Si $u$ est un minimiseur radial pour $1 / C_{G N}(p)$ et si $u_{n}(x):=u(x+n \mathrm{e})$, $\mathrm{e} \in \mathbb{S}^{d-1}$,

$$
\begin{array}{r}
\frac{1}{C_{C K N}(\vartheta(p, d), p, a)} \leq \frac{\left\||x|^{-a} \nabla u_{n}\right\|_{\mathrm{L}^{2}\left(\mathbb{R}^{d}\right)}^{2 \vartheta(p,)}\left\||x|^{-(a+1)} u_{n}\right\|_{\mathrm{L}^{2}\left(\mathbb{R}^{d}\right)}^{2(1-\vartheta(d))}}{\left\||x|^{-b} u_{n}\right\|_{\mathrm{L}^{p}\left(\mathbb{R}^{d}\right)}^{2}} \\
=\frac{1}{\mathrm{C}_{G N}(p)}\left(1+\mathcal{R} n^{-2}+O\left(n^{-4}\right)\right) .
\end{array}
$$

Donc, $\quad \frac{1}{C_{C K N}} \leq \frac{1}{C_{G N}}$.

Un résultat intéressant est que l'inégalité stricte $\frac{1}{C_{C K N}}<\frac{1}{C_{G N}}$ joue un rôle important pas seulement dans l'étude la compacité relative des suites minimisantes, mais aussi dans l'obtention d'estimations a priori pour les suites minimisantes.

\section{Symétrie et rupture de symétrie pour $\theta \in(0,1)$.}

Dans le cas $\theta \in(0,1)$ des résultats équivalents à ceux obtenus dans le cas $\theta=1$ peuvent être démontrés. En particulier, il existe une courbe qui sépare les régions de symétrie et de rupture de symétrie. Et cette courbe se situe "au dessus" de celle équivalente à celle de Felli-Schneider pour ce cas-ci. Une question intéressante est de nouveau celle de vérifier si ces deux courbes coïncident ou pas. Comme on verra ci-dessous, nous sommes capables de montrer qu'il y a des cas ou les deux courbes ne coïncident pas ; c'est-à-dire que pour certaines valeurs de $\theta \in(0,1), \Lambda$ et $p$ il existe des régions ou les fonctiones extrémales radiales sont stables (minima locaux), mais elles ne sont pas extrémales dans l'espace entier (ils ne sont pas des minima globaux) $!$

\section{Nouveaux résultats de rupture de symétrie.}

Nous savons que

$$
\frac{1}{C_{C K N}(\vartheta(p, d), p, \Lambda)} \leq \frac{1}{C_{G N}(p)}
$$

Si nous sommes capables de trouver une constante $\Lambda>0$ et une fonction $g$ telles que 


$$
\frac{1}{C_{G N}} \leq \mathcal{E}_{G N}[g]<\frac{1}{C_{C K N}^{*}(\vartheta(p, d), p, \Lambda)}
$$

alors,

$$
\frac{1}{C_{C K N}(\vartheta(p, d), p, \Lambda)} \leq \frac{1}{C_{G N}}<\frac{1}{C_{C K N}^{*}(\vartheta(p, d), p, \Lambda)},
$$

et nous aurons ainsi trouvé des cas de rupture de symétrie.

Un nouveau résultat de rupture de symétrie [11] (2010, J. Dolbeault, M.J. Esteban, G. Tarantello, A. Tertikas).

Soit $\mathrm{g}(x):=(2 \pi)^{-d / 4} \exp \left(-|x|^{2} / 4\right) . \quad$ Choisissons $\Lambda_{p, d}=\Lambda_{F S}(p(\theta, d), d)$

Il y a rupture de symétrie si

$$
\mathrm{L}(p, d):=\frac{\mathcal{E}_{G N}[g]}{\frac{1}{C_{C K N}^{*}\left(\vartheta(p, d), p, \Lambda_{p, d}\right)}}<1
$$

Nous avons le résultat suivant : pour tout $d \geq 3$, il existe un nombre $\tilde{p}(d) \in(2,2 d /(d-2))$ tel que pour tout $2<p<\tilde{p}(d), L(p, d)<1$. Par continuité, cela montre qu'il y a rupture de symétrie dans un voisinage du point $\left(\Lambda_{p, d}, p\right)$. Et un tel voisinage contient nécessairement un ensemble ouvert de points $(\Lambda, p)$ tels que $\Lambda_{F S}(p)<\Lambda$. Ceci prouve un résultat de rupture de symétrie dans une région ou les solutions extrémales radiales sont stables ! Mais dans le cas $\theta=1$ nous avons avancé dans la preuve de la conjecture énoncée ci-dessus : nous avons démontré la symétrie des fonctions extrémales pour une grande partie de la région $a<b<a+1$, $\Lambda<\Lambda_{F S}(p)$.

\section{Un nouveau résultat de symétrie [8] (2011, J. Dolbeault, M.J. Esteban. M. Loss).}

Pour $\theta=1$ et $d \geq 2$, utilisant une méthode qui utilise des inégalités de type Lieb-Thirring, l'inégalité de Beckner [2] sur la sphère unité et un argument de rigidité dû à Bidaut-Veron et Veron [3] pour les solutions positives de l'équation $-\Lambda_{g} u+\mu u=u^{q} \quad$ sur des variétés $(M, g)$, il est démontré dans [8] que pour tout $\Lambda \leq \tilde{\Lambda}(p)$, pour tout $p \in\left(2, \frac{2 d}{d-2}\right)$, il existe un "unique" minimiseur pour $(\mathrm{CKN})$, et il est symétrique, où

$$
\tilde{\Lambda}(p):=\frac{(d-1)(6-p)}{4(p-2)}<\Lambda_{F S}(p) .
$$


Idée de la preuve du résultat ci-dessus : Soit $L^{2}$ l'opérateur de LaplaceBeltrami sur $S^{d-1}$. Alors $-\Delta$ sur le cylindre devient $-\partial_{s}^{2}-L^{2}$.

THEOREME [8]. Soit $d \geq 2$ et soit $u$ une solution non-négative sur $\mathcal{C}=\mathbb{R} \times S^{d-1} \mathrm{de}$

$$
-\partial_{s}^{2} v-L^{2} v+\Lambda v=v^{p-1},
$$

et considérons la solution symérique $v_{*}$. Si

$$
\int_{\mathcal{C}}|v(s, \omega)|^{p} d s d \omega \leq \int_{\mathbb{R}}\left|v_{*}(s)\right|^{p} d s
$$

pour un $2<p<6$ satisfaisant $p \leq \frac{2 d}{d-2}$, et si $\Lambda \leq \tilde{\Lambda}(p)$, alors pour p.p. $\omega \in S^{d-1}$ et pour $s \in \mathbb{R}$, nous avons $v(s, \omega)=v_{*}(s-C), C$ étant une constante.

REMARQUE. Avec la normalisation définie ci-dessus,

$$
\frac{1}{C_{\Lambda, p}}=\inf \frac{\int_{\mathcal{C}}|\nabla v|^{2}+\Lambda v^{2} d x}{\left(\int_{\mathcal{C}}|v|^{p} d x\right)^{2 / p}}=\left(\int_{\mathcal{C}}|v(s, \omega)|^{p} d s d \omega\right)^{\frac{p-2}{p}} .
$$

REMARQUE. Nous choisissons $d \omega$ comme une mesure de probabilité sur $S^{d-1}$.

"Lieb-Thirring en 1-d". Ce résultat a été montré pour la première fois par J. B. Keller dans [15]. Voir aussi [16].

LEMMA. Soit $V=V(s)$ un potentiel non-négatif à valeurs réelles dans $\mathrm{L}^{\gamma+\frac{1}{2}}(\mathbb{R})$ pour $\gamma>1 / 2$ et soit $-\lambda_{1}(V)$ la valeur propre plus négative de l'opérateur Schrödinger $-\frac{d^{2}}{d s^{2}}-V$. Définissons

$$
c_{L T}(\gamma)=\frac{\pi^{-1 / 2}}{\gamma-1 / 2} \frac{\Gamma(\gamma+1)}{\Gamma(\gamma+1 / 2)}\left(\frac{\gamma-1 / 2}{\gamma+1 / 2}\right)^{\gamma+1 / 2} .
$$

Alors

$$
\lambda_{1}(V)^{\gamma} \leq c_{L T}(\gamma) \int_{\mathbb{R}} V^{\gamma+1 / 2}(s) d s,
$$

avec égalité si et seulement si, à translation et changement d'échelle près,

$$
V(s)=\frac{\gamma^{2}-1 / 4}{\cosh ^{2}(s)}=: V_{0}(s),
$$


et dans ce cas,

$$
\lambda_{1}\left(V_{0}\right)=(\gamma-1 / 2)^{2} .
$$

De plus, la fonction propre correspondante est :

$$
\psi_{\gamma}(s)=\pi^{-1 / 4}\left(\frac{\Gamma(\gamma)}{\Gamma(\gamma-1 / 2)}\right)^{1 / 2}[\cosh (s)]^{-\gamma+1 / 2} .
$$

Revenons maintenant à la démontration du théorème ci-dessus. Avec $V=v^{p-2}$, l'équation $-\Delta v+\Lambda v=v^{p-1}$ peut être vue comme une équation "linéaire" $-\Delta v-V v=-\Lambda v$.

Définissons $f(\omega):=\sqrt{\int_{\mathbb{R}}|v(s, \omega)|^{2} d s}$. Par le lemme de type "LiebThirring" cité ci-dessus, p.p. en $\omega$,

$$
\begin{gathered}
-\Lambda \int_{\mathcal{C}}|v(s, \omega)|^{2} d s d \omega=\int_{S^{d-1}} \int_{\mathbb{R}}\left(v_{s}^{2}-v^{p}\right) d s d \omega+\int_{\mathcal{C}}|L v|^{2} d s d \omega \\
=\int_{S^{d-1}} \int_{\mathbb{R}}\left(v_{s}^{2}-V v^{2}\right) d s d \omega+\int_{\mathcal{C}}|L v|^{2} d s d \omega=: \mathcal{F}[v] .
\end{gathered}
$$

Par convexité, nous avons,

$$
\mathcal{F}[v] \geq-c_{L T}(\gamma)^{1 / \gamma}\left(\int_{\mathbb{R}}|v(s, \omega)|^{p} d s\right)^{1 / \gamma}|f|^{2}+\int_{S^{d-1}}|L f|^{2} d \omega .
$$

Maintenant, définissant $D:=c_{L T}(\gamma)^{1 / \gamma}\left(\int_{\mathcal{C}} v^{p} d s d \omega\right)^{\frac{1}{\gamma}}$, et utilisant l'inégalité de Hölder, nous trouvons

$$
\mathcal{F}[v] \geq \int_{S^{d-1}}(L f)^{2} d \omega-D\left(\int_{S^{d-1}} f^{\frac{2 \gamma}{\gamma-1}} d \omega\right)^{\frac{\gamma-1}{\gamma}}=: \mathcal{E}[f] .
$$

L'inégalité généralisée de Poincaré sur la sphère dit que pour tout $q \in$ $\left(1, \frac{d+1}{d-3}\right]$

$$
\frac{q-1}{d-1} \int_{S^{d-1}}(L f)^{2} d \omega \geq\left(\int_{S^{d-1}} f^{q+1} d \omega\right)^{\frac{2}{q+1}}-\int_{S^{d-1}} f^{2} d \omega .
$$

Choisissant $q+1=\frac{2 \gamma}{\gamma-1}=2 \frac{p+2}{6-p}$,

$$
\mathcal{E}[f] \geq\left(\frac{d-1}{q-1}-D\right)\left(\int_{S^{d-1}} f^{q+1} d \omega\right)^{\frac{2}{q+1}}-\frac{d-1}{q-1} \int_{S^{d-1}} f^{2} d \omega .
$$


Ce calcul est justifié, car $q \leq \frac{d+1}{d-3}$ est équivalent à $p \leq \frac{2 d}{d-2}$.

Utilisant le fait que $d \omega$ est une mesure de probabilité, par l'inégalité de Hölder, nous trouvons

$$
\left(\int_{S^{d-1}} f^{q+1} d \omega\right)^{\frac{2}{q+1}} \geq \int_{S^{d-1}} f^{2} d \omega .
$$

Donc, si $D \leq \frac{d-1}{q-1}$, et si $\Lambda \leq \tilde{\Lambda}(p)$, nous trouvons

$$
-\Lambda \int_{S^{d-1}} f^{2} d \omega \geq \mathcal{E}[f] \geq-D \int_{S^{d-1}} f^{2} d \omega \geq-\Lambda \int_{S^{d-1}} f^{2} d \omega
$$

\section{CONCLUSION.}

Pour $\theta=1$, la zone de rupture de symmétrie coïncide "presque" avec la zone d'instabilité du minimiseur symétrique, puisque la différence entre $\tilde{\Lambda}$ et $\Lambda_{F S}$ est petite, surtout lorque $d$ croît. Mais nous avons quand même que $\tilde{\Lambda}$ est toujours plus petite que $\Lambda_{F S}$, strictement.

MAIS quand $\theta \in\left(0, \theta_{0}\right), \theta_{0}<1$, il y a rupture de symétrie en dehors de la zone de Felli-Schneider, c'est-à-dire, dans des endroits où le minimiseur symétrique est stable!

\section{References}

[1] T. Aubin, Problèmes isopérimétriques et espaces de Sobolev, J. Differential Geometry, 11 (1976), pp. 573-598.

[2] W. Beckner, Sharp Sobolev inequalities on the sphere and the MoserTrudinger inequality, Ann. of Math. (2), 138 (1993), pp. 213-242.

[3] M.-F. Bidaut-VÉron And L. VÉron, Nonlinear elliptic equations on compact Riemannian manifolds and asymptotics of Emden equations, Invent. Math., 106 (1991), pp. 489-539.

[4] L. Caffarelli, R. Kohn, and L. Nirenberg, First order interpolation inequalities with weights, Compositio Math., 53 (1984), pp. 259275. 
[5] F. Catrina And Z.-Q. WAng, On the Caffarelli-Kohn-Nirenberg inequalities: sharp constants, existence (and nonexistence), and symmetry of extremal functions, Comm. Pure Appl. Math., 54 (2001), pp. 229 258.

[6] K. S. Chou And C. W. Chu, On the best constant for a weighted Sobolev-Hardy inequality, J. London Math. Soc. (2), 48 (1993), pp. 137151.

[7] M. Del Pino, J. Dolbeault, S. Filippas, and A. Tertikas, $A$ logarithmic Hardy inequality, Journal of Functional Analysis, 259 (2010), pp. $2045-2072$.

[8] J. Dolbeault, M. J. Esteban, And M. Loss, Symmetry of extremals of functional inequalities via spectral estimates for linear operators, To appear in J. Math. Physics, (2012).

[9] J. Dolbeault, M. J. Esteban, M. Loss, and G. Tarantello, On the symmetry of extremals for the Caffarelli-Kohn-Nirenberg inequalities, Adv. Nonlinear Stud., 9 (2009), pp. 713-726.

[10] J. Dolbeault, M. J. Esteban, and G. Tarantello, The role of Onofri type inequalities in the symmetry properties of extremals for Caffarelli-Kohn-Nirenberg inequalities, in two space dimensions, Ann. Sc. Norm. Super. Pisa Cl. Sci. (5), 7 (2008), pp. 313-341.

[11] J. Dolbeault, M. J. Esteban, G. Tarantello, and A. TerTIKAS, Radial symmetry and symmetry breaking for some interpolation inequalities, To appear in Calculus of Variations and PDE, (2011).

[12] V. Felli And M. Schneider, Perturbation results of critical elliptic equations of Caffarelli-Kohn-Nirenberg type, J. Differential Equations, 191 (2003), pp. 121-142.

[13] V. Glaser, A. Martin, H. Grosse, and W. Thirring, A family of optimal conditions for the absence of bound states in a potential, Essays in Honor of Valentine Bargmann, E. Lieb, B. Simon, A. Wightman Eds. Princeton University Press, 1976, pp. 169-194.

[14] T. Horiuchi, Best constant in weighted Sobolev inequality with weights being powers of distance from the origin, J. Inequal. Appl., 1 (1997), pp. 275-292. 
[15] J. B. KELLER, Lower bounds and isoperimetric inequalities for eigenvalues of the Schrödinger equation, J. Mathematical Phys., 2 (1961), pp. 262-266.

[16] E. Lieb And W. Thirring, Inequalities for the moments of the eigenvalues of the Schrödinger Hamiltonian and their relation to Sobolev inequalities, Essays in Honor of Valentine Bargmann, E. Lieb, B. Simon, A. Wightman Eds. Princeton University Press, 1976, pp. 269-303.

[17] E. H. LiEB, Sharp constants in the Hardy-Littlewood-Sobolev and related inequalities, Ann. of Math. (2), 118 (1983), pp. 349-374.

[18] C.-S. Lin AND Z.-Q. WANG, Erratum to: "Symmetry of extremal functions for the Caffarelli-Kohn-Nirenberg inequalities" [Proc. Amer. Math. Soc. 132 (2004), no. 6, 1685-1691], Proc. Amer. Math. Soc., 132 (2004), p. 2183.

[19] P.-L. Lions, The concentration-compactness principle in the calculus of variations. The locally compact case. I, Ann. Inst. H. Poincaré Anal. Non Linéaire, 1 (1984), pp. 109-145.

[20] - The concentration-compactness principle in the calculus of variations. The locally compact case. II, Ann. Inst. H. Poincaré Anal. Non Linéaire, 1 (1984), pp. 223-283.

[21] G. Talenti, Best constant in Sobolev inequality, Ann. Mat. Pura Appl. (4), 110 (1976), pp. 353-372. 